Глушак А.В. Критерий единственности решения граничных задач для абстрактного уравнения Эйлера-Пуассона-Дарбу на конечном интервале. Математ. заметки. 2021. Т. 109, вып. 6. С. 821-831.

УДК 517.983.23

\title{
Критерий единственности решения граничных задач для абстрактного уравнения Эйлера-Пуассона-Дарбу на конечном интервале
}

\author{
Глушак А.В.
}

Пусть $E$ - комплексное банахово пространство и $A-$ линейный замкнутый оператор в $E$, область определения $D(A) \subset E$ которого не обязательно плотна в $E$. Мы будем изучать граничные задачи на конечном интервале $0<t<1$, поскольку общий случай изменения $0<t<T$ сводится к рассматриваемому заменой переменной $t$ на $t / T$. Постановка граничных условий для уравнения Эйлера-Пуассона-Дарбу

$$
u^{\prime \prime}(t)+\frac{k}{t} u^{\prime}(t)=A u(t), \quad 0<t<1
$$

в силу сингулярности уравнения в точке $t=0$ зависит от параметра $k \in \mathbb{R}$ и эти условия будут приведены далее. Граничные условия в точке $t=1$ для всех рассматриваемых случаев изменения параметра $k$ одинаковы и имеют вид

$$
\alpha u(1)+\beta u^{\prime}(1)=u_{1}, \quad u_{1} \in E .
$$

где $\alpha, \beta \in \mathbb{R}, \alpha^{2}+\beta^{2}>0$.

Граничные задачи для уравнения (1), вообще говоря, не являются корректными, но необходимость решать некорректные задачи в настоящее время является общепризнанной (см. введение в [1], [2], [3] и имеющуюся в них обширную библиографию). Во второй главе монографии [1] исследована корректность общих граничных задач для дифференциальнооператорного уравнения первого порядка и для абстрактного несингулярного уравнения второго порядка (случай $k=0$ в уравнении (1)).

Мы приведем постановку различных граничных задач для уравнения (1) в зависимости от параметра $k \in \mathbb{R}$ и установим соответствующие критерии единственности их решений. Будет показано, что единственность решения зависит лишь от расположения на комплексной плоскости $\mathbb{C}$ собственных значений оператора $A$ и связана с распределением нулей некоторых аналитических функций. Поскольку на оператор $A$ наложены весьма общие условия, то в этой работе вопросов разрешимости граничных задач мы, естественно, не касаемся. Укажем лишь, что результаты о разрешимости граничных задач в полупространстве для уравнения Эйлера-Пуассона-Дарбу в частных производных приведены в [4], а граничные задачи на полуоси для абстрактных сингулярных уравнений исследовались в $[5,6]$.

Важную роль при установлении критерия единственности будет играть задача на собственные значения для обыкновенного дифференциального уравнения

$$
v^{\prime \prime}(t)+\frac{k}{t} v^{\prime}(t)=\lambda v(t), \quad 0<t<1 .
$$


Структура общего решения этого дифференциального уравнения при всех значениях параметра $k \in \mathbb{R}$ указана в [7] и нужные в дальнейшем результаты мы будем напоминать по мере их использования.

1. Случай $k<1$. Условие Дирихле при $\boldsymbol{t}=\mathbf{0}$. Рассмотрим задачу определения функции $u(t) \in C([0,1], E) \bigcap C^{2}((0,1], E)$, принадлежащей $D(A)$ при $t \in(0,1)$, удовлетворяющей уравнению Эйлера-Пуассона-Дарбу (1), условию (2), а также граничному условию Дирихле

$$
u(0)=u_{0}, \quad u_{0} \in E .
$$

Исследование единствености решения задачи (1) - (3) сводится к вопросу об отсутствии у уравнения (1) нетривиальных решений $u(t)$, удовлетворяющих нулевым условиям

$$
\begin{gathered}
u(0)=0, \\
\alpha u(1)+\beta u^{\prime}(1)=0,
\end{gathered}
$$

поскольку нулевое решение $u(t) \equiv 0$ у этой задачи всегда имеется.

Нетривиальные решения $u(t)$ однородной задачи $(1),(4),(5)$ будем искать методом разделения переменных в виде $u(t)=v(t) h$, где $v(t) \in C[0,1] \bigcap C^{2}(0,1]$ - ненулевая скалярная комплекснозначная функция, $h \in D(A), h \neq 0$.

Подставляя $u(t)=v(t) g$ в задачу $(1),(4),(5)$, будем иметь

$$
v^{\prime \prime}(t) h+\frac{k}{t} v^{\prime}(t) h=v(t) A h,
$$

и условия

$$
\begin{gathered}
v(0)=0, \\
\alpha v(1)+\beta v^{\prime}(1)=0 .
\end{gathered}
$$

Из уравнения (6) вытекает равенство

$$
A h=\frac{v^{\prime \prime}(t)+k / t v^{\prime}(t)}{v(t)} h,
$$

которое должно выполняться на множестве $\{t \in(0,1): v(t) \neq 0\}$.

Очевидно, равенство (9) может быть справедливым только, если

$$
A h=\lambda h
$$

с некоторой постоянной $\lambda \in \mathbb{C}$.

Таким образом, в силу (10), элемент $h \in D(A), h \neq 0$ должен быть собственным вектором оператора $A$ с собственным значением $\lambda \in \mathbb{C}$, при этом уравнение (6) превратится в уравнение

$$
v^{\prime \prime}(t)+\frac{k}{t} v^{\prime}(t)=\lambda v(t) .
$$

В работе [7] установлено, что общее решение обыкновенного дифференциального уравнения (11) представимо в виде

$$
v(t)=c_{1} Y_{k}(t ; \lambda)+c_{2} t^{1-k} Y_{2-k}(t ; \lambda), \quad c_{1}, c_{2} \in \mathbb{R},
$$

где $Y_{k}(t ; A)$ - построенный в [7] разрешающий оператор задачи Коши для уравнения (1), $Y_{k}(0 ; A)=I$. Конкретные представления для $Y_{k}(t ; \lambda)$ и $Y_{2-k}(t ; \lambda)$ будут указаны по ходу доказательства. 
Поэтому удовлетворяющее начальному условию (7) решение уравнения (11) имеет вид

$$
v(t)=t^{1-k} Y_{2-k}(t ; \lambda),
$$

где при $k<1$

$$
Y_{2-k}(t ; \lambda)=\Gamma(3 / 2-k / 2)(t \sqrt{\lambda} / 2)^{k / 2-1 / 2} I_{1 / 2-k / 2}(t \sqrt{\lambda}),
$$

$\Gamma(\cdot)$ - гамма-функция Эйлера, $I_{\nu}(\cdot)$ - модифицированная функция Бесселя. Функцию $Y_{2-k}(t ; \lambda)$ также называют нормированной функцией Бесселя и обозначают $j_{1 / 2-k / 2}(t \sqrt{\lambda})$.

Для нахождения подходящих собственных значений $\lambda \in \mathbb{C}$ осталось воспользоваться граничным условием (8), подставляя в которое функцию (13), получим трансцендентное уравнение

$$
(\alpha+\beta(1-k)) Y_{2-k}(1 ; \lambda)+\beta Y_{2-k}^{\prime}(1 ; \lambda)=0 .
$$

Обозначив $\sqrt{\lambda}=i \mu$ и учитывая представление (14), в терминах функции Бесселя первого рода $J_{\nu}(\cdot)$ уравнение (15) запишем в виде

$$
\frac{(\alpha+\beta(1-k) / 2) J_{1 / 2-k / 2}(\mu)+\beta \mu J_{1 / 2-k / 2}^{\prime}(\mu)}{\mu^{1 / 2-k / 2}}=0 .
$$

Как известно (см. п. 18.3 [8]), уравнение (16) имеет бесконечное множество расположенных в порядке возрастания положительных корней $\mu_{m}, m \in \mathbb{N}$. Подставляя $\lambda_{m}=-\mu_{m}^{2}$ в (13) получим функции

$$
v_{m}(t)=t^{1-k}\left(Y_{2-k}\left(t ; \lambda_{m}\right), \quad m \in \mathbb{N},\right.
$$

которые являются нетривиальными решениями задачи $(11),(7),(8)$, при этом соотношение (10) превратится в уравнения для нахождения $h_{m} \neq 0$

$$
A h_{m}=\lambda_{m} h_{m}, \quad m \in \mathbb{N} .
$$

Предположим далее, что число $\lambda_{m}$ при некотором $m \in \mathbb{N}$ является собственным значением оператора $A$ с собственным вектором $h_{m} \neq 0$. Тогда мы определим нетривиальное решение однородной задачи (1), (4), (5) следующего вида

$$
u_{m}(t)=t^{1-k} Y_{2-k}\left(t ; \lambda_{m}\right) h_{m} .
$$

Сформулируем теперь критерий единственности решения граничной задачи (1) - (3).

Теорема 1. Пусть $k<1$ и $A$ - линейный замкнутый оператор в $E$. Предположим, что граничная задача $(1)-(3)$ имеет решение $u(t)$. Для того, чтобы это решение было единственным, необходимо и достаточно, чтобы ни один нуль $\lambda_{m}, m \in \mathbb{N}$ функции

$$
\Upsilon_{k}^{\alpha, \beta}(\lambda)=(\alpha+\beta(1-k)) Y_{2-k}(1 ; \lambda)+\beta Y_{2-k}^{\prime}(1 ; \lambda),
$$

не являлся бы собственным значением оператора $A$.

Доказательство. Как уже отмечалось ранее, исследование единственности решения задачи (1) - (3) сводится к вопросу об отсутствии у уравнения (1) нетривиальных решений $u(t)$, удовлетворяющих нулевым условиям (4), (5).

Необходимость. Предположим противное, пусть некоторый нуль $\lambda_{m}, m \in \mathbb{N}$ из счетного множества $\Lambda_{k}^{\alpha, \beta}$ нулей функции $\Upsilon_{k}^{\alpha, \beta}(\lambda)$ является собственным значением оператора $A$ 
с собственным вектором $h_{m} \neq 0$. Тогда определяемая равенством (18) функция $u_{m}(t)$ служит нетривиальным решением однородной обратной задачи $(1),(4),(5)$, что противоречит единственности решения этой задачи, и необходимость доказана.

Докажем теперь достаточность. Предположим, что ни одно число $\lambda_{m}$ из счетного множества $\Lambda_{k}^{\alpha, \beta}$ нулей, определяемой равенством (19) функции $\Upsilon_{k}^{\alpha, \beta}(\lambda)$, не является собственным значением оператора $A$, и пусть $u(t)$ - некоторое решение однородной граничной задачи $(1),(4),(5)$. Покажем, что в этом случае $u(t) \equiv 0$.

Введем в рассмотрение функцию $U(\lambda)$ переменной $\lambda \in \mathbb{C}$ со значениями в банаховом пространстве $E$

$$
U(\lambda)=\int_{0}^{1} t Y_{2-k}(t ; \lambda) u(t) d t
$$

где скалярная функция $Y_{2-k}(t ; \lambda)$ определена равенством $(14)$, является решением уравнения (11) и удовлетворяет условиям $Y_{2-k}(0 ; \lambda)=I, Y_{2-k}^{\prime}(0 ; \lambda)=0$.

Учитывая замкнутость оператора $A$ и равенство $(1)$, вычислим $A U(\lambda)$. После двукратного интегрирования по частям будем иметь

$$
\begin{gathered}
A U(\lambda)=\int_{0}^{1} t Y_{2-k}(t ; \lambda) A u(t) d t=\int_{0}^{1} t Y_{2-k}(t ; \lambda)\left(u^{\prime \prime}(t)+\frac{k}{t} u^{\prime}(t)\right) d t= \\
=\left.t Y_{2-k}(t ; \lambda) u^{\prime}(t)\right|_{0} ^{1}+\int_{0}^{1}\left((k-1) Y_{2-k}(t ; \lambda)-t Y_{2-k}^{\prime}(t ; \lambda)\right) u^{\prime}(t) d t= \\
=Y_{2-k}(1 ; \lambda) u^{\prime}(1)+\left.\left((k-1) Y_{2-k}(t ; \lambda)-t Y_{2-k}^{\prime}(t ; \lambda)\right) u(t)\right|_{0} ^{1}+ \\
\quad+\int_{0}^{1} t\left(Y_{2-k}^{\prime \prime}(t ; \lambda)+\frac{2-k}{t} Y_{2-k}^{\prime}(t ; \lambda)\right) u(t) d t= \\
=Y_{2-k}(1 ; \lambda) u^{\prime}(1)+\left((k-1) Y_{2-k}(1 ; \lambda)-Y_{2-k}^{\prime}(1 ; \lambda)\right) u(1)+\lambda U(\lambda) .
\end{gathered}
$$

Пусть в граничном условии (5) коэффициент $\beta \neq 0$. Тогда равенство (21) примет вид

$$
(\lambda I-A) U(\lambda)=\frac{1}{\beta}\left((\alpha+\beta(1-k)) Y_{2-k}(1 ; \lambda)+\beta Y_{2-k}^{\prime}(1 ; \lambda)\right) u(1) .
$$

Таким образом, для всех чисел $\lambda_{m} \in \Lambda_{k}^{\alpha, \beta}$ из счетного множества нулей определяемой равенством (19) функции $\Upsilon_{k}^{\alpha, \beta}(\lambda)$, из равенства (22) вытекает соотношение

$$
A U\left(\lambda_{m}\right)=\lambda_{m} U\left(\lambda_{m}\right) .
$$

По предположению, ни одно из таких чисел $\lambda_{m}$ не является собственным значением оператора $A$. Но тогда все значения $U\left(\lambda_{m}\right)$ должны равняться нулю

$$
U\left(\lambda_{m}\right)=0, m \in \mathbb{N} .
$$

Пусть $\mu_{m}, m \in \mathbb{N}$ - расположенные в порядке возрастания положительные корни уравнения (16) и $\left(i \mu_{m}\right)^{2}=\lambda_{m}$. Тогда равенства (23) принимают вид

$$
U_{m}=\int_{0}^{1} t^{k / 2+1 / 2} J_{1 / 2-k / 2}\left(t \mu_{m}\right) u(t) d t=0, m \in \mathbb{N} .
$$


Применив к определяемым равенством (24) векторным коэффициентам $U_{m}$ линейный непрерывный функционал $f \in E^{*}$, получим скалярную функцию $\varphi(t)=f\left(t^{k / 2-1 / 2} u(t)\right)$, удовлетворяющую условиям

$$
f\left(U_{m}\right)=\int_{0}^{1} t J_{1 / 2-k / 2}\left(t \mu_{m}\right) \varphi(t) d t=0, \quad m \in \mathbb{N} .
$$

Дальнейшие рассуждения зависят от коэффициентов $\alpha, \beta, k$.

а) Если в граничном условии (5) коэффициенты $\alpha, \beta, k$ таковы, что

$$
\frac{\alpha}{\beta}+1-k>0
$$

то с точностью до множителя скалярные коэффициенты $f\left(U_{m}\right)$ являются коэффициентами ряда Дини (см. п. $18.3[8])$

$$
\sum_{m=1}^{\infty} b_{m} J_{1 / 2-k / 2}\left(t \mu_{m}\right)
$$

разложения функции $\varphi(t)$ по функциям $J_{1 / 2-k / 2}\left(t \mu_{m}\right)$.

Функция $\varphi(t)$ вполне определена своими коэффициентами ряда Дини независимо от того, сходится этот ряд или нет, следовательно, $\varphi(t) \equiv 0,0 \leq t \leq 1$. Поскольку выбор функционала $f \in E^{*}$ был произвольным, то и $u(t) \equiv 0,0 \leq t \leq 1$.

b) Если

$$
\frac{\alpha}{\beta}+1-k=0
$$

то к разложению Дини (27) следует дополнительно добавить (см. п. 18.3 [8]) слагаемое $b_{0} t^{1 / 2-k / 2}$, где $b_{0}=2 f\left(U_{0}\right)$, а $U_{0}$ определяется формулой $(24)$ по значению $\mu_{0}=0$.

И в этом случае, учитывая равенство (28) и граничное условие (5), получим

$$
\varphi(t)=b_{0} t^{1 / 2-k / 2}, \quad \alpha \varphi(1)+\beta \varphi^{\prime}(1)=b_{0} \frac{\beta(k-1)}{2}=0 .
$$

Поскольку $\beta \neq 0, k<1$, то $b_{0}=0$ и $\varphi(t) \equiv 0, u(t) \equiv 0,0 \leq t \leq 1$.

c) Пусть

$$
\frac{\alpha}{\beta}+1-k<0
$$

В этом случае к разложению Дини (27) следует дополнительно добавить (см. п. 18.3 [8]) слагаемое $b_{0} I_{1 / 2-k / 2}\left(t \mu_{0}\right)$, где $\pm i \mu_{0}\left(\mu_{0}>0\right)$ - два чисто мнимых корня уравнения (16) и тогда учитывая граничное условие $(5)$, получим

$$
\begin{gathered}
\varphi(t)=b_{0} I_{1 / 2-k / 2}\left(t \mu_{0}\right), \\
\alpha \varphi(1)+\beta \varphi^{\prime}(1)=b_{0}\left(\alpha I_{1 / 2-k / 2}\left(\mu_{0}\right)+\beta \mu_{0} I_{1 / 2-k / 2}^{\prime}\left(\mu_{0}\right)\right)=b_{0} \frac{\beta(k-1)}{2} I_{1 / 2-k / 2}\left(\mu_{0}\right)=0 .
\end{gathered}
$$

Модифицированная функция Бесселя $I_{1 / 2-k / 2}\left(\mu_{0}\right)$ при действительных значениях $\mu_{0}$ в нуль не обращается, поэтому, опять-таки $b_{0}=0$ и $\varphi(t) \equiv 0, u(t) \equiv 0,0 \leq t \leq 1$.

Тем самым установлено, что решение $u(t)$ однородной задачи $(1),(4),(5)$, а, следовательно, и задачи (1) - (3), может быть только нулевым. 
d) Если, наконец, $\beta=0$, то доказательство проводится аналогично рассуждениям пункта а), при этом вместо ряда Дини (27), где $\mu_{m}$ - корни уравнения (16), используется ряд Фурье-Бесселя, построенный по нулям функции $J_{1 / 2-k / 2}(\mu)$.

Теорема доказана.

Нули функции $\Upsilon_{k}^{\alpha, \beta}(\lambda)$ при $k<1$ удобно определять равенством $\lambda_{m}=-\mu_{m}^{2}$, где $\mu_{m}$ корни уравнения (16). Например, если $k=0$, то уравнение (16) принимает вид

$$
\alpha \frac{\sin \mu}{\mu}+\beta \cos \mu=0 .
$$

В некоторых случаях корни этого уравнения явно вычисляются, в частности, при $\alpha=0$ имеем $\mu_{m}=\pi / 2+\pi m, m \in \mathbb{N}$, поэтому $\Lambda_{0}^{0, \beta}=\left\{-(\pi / 2+\pi m)^{2}, m \in \mathbb{N}\right\}$, а если $\beta=0$, то $\mu_{m}=\pi m, m \in \mathbb{N}$, a $\Lambda_{0}^{\alpha, 0}=\left\{-(\pi m)^{2}, m \in \mathbb{N}\right\}$.

Таким образом, чтобы решить вопрос о единственности решения рассматриваемой граничной задачи, следует определить собственные значения оператора $A$ и выяснить их принадлежность множеству $\Lambda_{k}^{\alpha, \beta}$ нулей функции $\Upsilon_{k}^{\alpha, \beta}(\lambda)$. Многочисленные примеры нахождения собственных значений для дифференциальных операторов $A$, действующих по пространственным переменным можно найти, например, в гл. 2 [9], и в каждом конкретном случае их следует сравнить с нулями функции $\Upsilon_{k}^{\alpha, \beta}(\lambda)$.

Мы рассмотрим пример действующего по пространственной переменной $x$ сингулярного оператора $A$. Для заданного на множестве функций

$$
D(A)=H^{2}(0,1) \bigcap H_{0}^{1}(0,1) \subset E=L_{2}(0,1)
$$

дифференциального оператора Бесселя $A=B_{q, x}$, где

$$
B_{q, x}=\frac{d^{2}}{d x^{2}}+\frac{q}{x} \frac{d}{d x}, \quad q>0,
$$

единственность решения рассматриваемых граничных задач сводится к исследованию расположения нулей функции $I_{q / 2-1 / 2}(\sqrt{z})$, которые являются собственными значениями оператора $B_{q, x}$, и нулей определяемой равенством (19) функции $\Upsilon_{k}^{\alpha, \beta}(\lambda)$.

В частности, при $\beta=0, k<1$ предстоит исследовать расположения нулей функций $I_{q / 2-1 / 2}(\sqrt{z})$ и $I_{1 / 2-k / 2}(\sqrt{\lambda})$. В зависимости от параметров $k$ и $q$, указанные функции Бесселя могут иметь, а могут и не иметь общих нулей, расположенных на $(-\infty, 0)$, поэтому единственность решения граничных задач может иметь место, а может и нарушаться. Подробнее о расположении нулей функций Бесселя см., например, в п.2 работы [10]. Отметим также, что важную роль при исследовании единственности играют и промежутки изменения переменных $0<t<T$ и $0<x<l$, поскольку при этом нули каждой из функций Бесселя меняют свое положение. Аналогичные факты при решении задачи Дирихле для гиперболических уравнений в частных производных установлены ранее в [11].

В случаях $A=-B_{q, x}$ или $A=i B_{q, x}$, где $i$ - мнимая единица, собственные значения оператора $A$ лежат либо на $(0,+\infty)$, либо на мнимой оси и не попадают на $(-\infty, 0)$, поэтому соответствующие граничные задачи имеют единственное решение.

2. Случай $\boldsymbol{k} \geq \mathbf{0}$. Весовое условие Неймана при $\boldsymbol{t}=\mathbf{0}$. Как видно из представления (12) для общего решения уравнения (11), уравнение (1) может иметь и неограниченные при $t=0$ решения, если вместо условия Дирихле задавать весовое условие Неймана. Предложенный в п.1 метод доказательства единственности использует разложения в ряды Дини и Фурье-Бесселя, поэтому постановка граничного условия в точке $t=0$ должна 
быть такой, чтобы у общего решения обыкновенного дифференциального уравнения (11) только одна из постоянных $c_{1}$ или $c_{2}$ обратилась в нуль. Первая такая возможность реализована в п.1 $\left(c_{1}=0, c_{2}=1\right)$. Еще одной возможностью является весовое граничное условие Неймана вида

$$
\lim _{t \rightarrow 0+} t^{k} u^{\prime}(t)=u_{2} \in E .
$$

Удовлетворяющее однородному начальному условию (30) решение уравнения (11) имеет вид $\left(c_{1}=1, c_{2}=0\right)$

$$
v(t)=Y_{k}(t ; \lambda)
$$

где при $k \geq 0$

$$
Y_{k}(t ; \lambda)=\Gamma(k / 2+1 / 2)(t \sqrt{\lambda} / 2)^{1 / 2-k / 2} I_{k / 2-1 / 2}(t \sqrt{\lambda}) .
$$

Дальнейшая схема установления критерия единственности аналогична п.1, при этом вместо соотношений (15), (16), (18), (20) следует использовать соответственно следующие равенства:

$$
\begin{gathered}
\alpha Y_{k}(1 ; \lambda)+\beta Y_{k}^{\prime}(1 ; \lambda)=0, \\
\frac{(\alpha+\beta(1-k) / 2) J_{k / 2-1 / 2}(\mu)+\beta \mu J_{k / 2-1 / 2}^{\prime}(\mu)}{\mu^{k / 2-1 / 2}}=0, \\
u_{m}(t)=Y_{k}\left(t ; \lambda_{m}\right) h_{m}, \\
U(\lambda)=\int_{0}^{1} t^{k} Y_{k}(t ; \lambda) u(t) d t .
\end{gathered}
$$

В результате мы приходим к следующему критерию единственности.

Теорема 2. Пусть $k \geq 0$ и $A$ - линейный замкнутый оператор в $E$. Предположим, что граничная задача $(1),(2),(30)$ имеет решение $u(t)$. Для того, чтобы это решение было единственным, необходимо и достаточно, чтобы ни один нуль $\lambda_{m}, m \in \mathbb{N}$ функции

$$
\Upsilon_{k}^{\alpha, \beta}(\lambda)=\alpha Y_{k}(1 ; \lambda)+\beta Y_{k}^{\prime}(1 ; \lambda)
$$

не являлся бы собственным значением оператора $A$.

Нули задаваемой равенством (33) функции $\Upsilon_{k}^{\alpha, \beta}(\lambda)$ удобно определять равенством $\lambda_{m}=$ $-\mu_{m}^{2}$, где $\mu_{m}-$ корни уравнения (32).

Если $k=0$, то уравнение (32) принимает вид

$$
\alpha \cos \mu-\beta \mu \sin \mu=0 .
$$

$\mathrm{B}$ частности, при $\beta=0$ имеем $\mu_{m}=\pi / 2+\pi m, \lambda_{m}=-\mu_{m}^{2}, m \in \mathbb{N}$, а если $\alpha=0$, то $\mu_{0}=\lambda_{0}=0, \mu_{m}=\pi m, \lambda_{m}=-(\pi m)^{2}, m \in \mathbb{N}$.

Заметим, что наличие нуля $\lambda_{0}=0$ у функции $\Upsilon_{k}^{\alpha, \beta}(\lambda)$ при установлении однозначной разрешимости граничных задач приводит к необходимости существования обратного оператора $A^{-1}$.

Если $k=2$, то уравнение (32) принимает вид

$$
\frac{(\alpha-\beta) \sin \mu+\beta \mu \cos \mu}{\mu}=0 .
$$

$\mathrm{B}$ частности, при $\beta=0$ имеем $\mu_{m}=\pi m, \lambda_{m}=-(\pi m)^{2}, m \in \mathbb{N}$, а если $\alpha=\beta \neq 0$, то $\mu_{m}=\pi / 2+\pi m, \lambda_{m}=-\mu_{m}^{2}, m \in \mathbb{N}$. 
3. Случай $0 \leq k<\mathbf{1}$. Для указанных значений параметра $k$ одновременно справедливы и теорема 1 с условием Дирихле при $t=0$, и теорема 2 с весовым условием Неймана при $t=0$.

4. Случай $-1<k<0$. Весовое условие Неймана при $t=0$. Условие Дирихле или Неймана при $t=1$. Наряду с условием Дирихле при $t=0$, которое было рассмотрено в п.1, рассмотрим и весовое условие Неймана. В отличие от п.2 при отрицательных значениях индекса $-1<k<0$ для функции $v(t)=Y_{k}(t ; \lambda)$ вместо равенства (31) следует использовать (см. [7]) другое представление, а именно:

$$
\begin{gathered}
v(t)=Y_{k}(t ; \lambda)=Y_{k+2}(t ; \lambda)+\frac{t}{k+1} Y_{k+2}^{\prime}(t ; \lambda)= \\
=\frac{\Gamma(k / 2+3 / 2)}{(t \sqrt{\lambda})^{k / 2+1 / 2}}\left(\frac{1}{2} I_{k / 2+1 / 2}(t \sqrt{\lambda})+\frac{t \sqrt{\lambda}}{k+1} I_{k / 2+1 / 2}^{\prime}(t \sqrt{\lambda})\right) .
\end{gathered}
$$

Для нахождения подходящих собственных значений $\lambda \in \mathbb{C}$ воспользуемся граничным условием (8), подставляя в которое функцию (34), получим трансцендентное уравнение

$$
\left(\alpha+\frac{\beta \lambda}{k+1}\right) Y_{k+2}(1 ; \lambda)+\frac{\alpha}{k+1} Y_{k+2}^{\prime}(1 ; \lambda)=0
$$

или

$$
(\sqrt{\lambda})^{-k / 2-1 / 2}\left(\left(\frac{\alpha}{2}+\frac{\beta \lambda}{k+1}\right) I_{k / 2+1 / 2}(\sqrt{\lambda})+\frac{\alpha \sqrt{\lambda}}{k+1} I_{k / 2+1 / 2}^{\prime}(\sqrt{\lambda})\right)=0 .
$$

Ввиду наличия множителя $\lambda$ перед функцией $I_{k / 2+1 / 2}(\sqrt{\lambda})$ корни уравнение $(35)$, вообще говоря, не связаны с разложениями функций в ряды Фурье-Бесселя и Дини, за исключением случаев $\alpha=0$ или $\beta=0$. Именно эти случаи мы далее и рассмотрим.

4.1. Условие Неймана при $\boldsymbol{t}=\mathbf{1}$. Если $\alpha=0$, то граничное условие $(2)$ превращается в условие Неймана при $t=1$

$$
u^{\prime}(1)=u_{1}, \quad u_{1} \in E,
$$

а уравнение (35) принимает вид

$$
\lambda^{3 / 4-k / 4} I_{k / 2+1 / 2}(\sqrt{\lambda})=0
$$

или при $\lambda=-\mu^{2}$

$$
\mu^{3 / 2-k / 2} J_{k / 2+1 / 2}(\mu)=0 .
$$

Обозначим корни уравнения (37) через $\mu_{0}=0, \mu_{m}>0, m \in \mathbb{N}, \lambda_{m}=-\mu_{m}^{2}$.

Дальнейшая схема установления критерия единственности аналогична доказательству теоремы $1(\mathrm{~d})$ или 1(a). В результате получим следующий критерий единственности.

Теорема 3. Пусть $-1<k<0$ и $A-$ линейный замкнутый оператор в $E$. Предположим, что граничная задача $(1),(30),(36)$ имеет решение $u(t)$. Для того, чтобы это решение было единственным, необходимо и достаточно, чтобы ни один нуль $\lambda_{m}, m=0,1,2 \ldots$ функции

$$
\Upsilon_{k}^{0, \beta}(\lambda)=\lambda Y_{k+2}(1 ; \lambda)
$$

не являлся бы собственным значением оператора $A$.

4.2. Условие Дирихле при $\boldsymbol{t}=\mathbf{1}$. Если $\beta=0$, то граничное условие $(2)$ превращается в условие Дирихле при $t=1$

$$
u(1)=u_{1}, \quad u_{1} \in E,
$$


а уравнение (35) принимает вид

$$
\frac{(k+1) I_{k / 2+1 / 2}(\sqrt{\lambda})+2 \sqrt{\lambda} I_{k / 2+1 / 2}^{\prime}(\sqrt{\lambda})}{\lambda^{k / 4+1 / 4}}=0,
$$

или при $\lambda=-\mu^{2}$

$$
\frac{(k+1) J_{k / 2+1 / 2}(\mu)+2 \mu J_{k / 2+1 / 2}^{\prime}(\mu)}{\mu^{k / 2+1 / 2}}=0 .
$$

Обозначим положительные корни уравнения (39) через $\mu_{m}, m \in \mathbb{N}, \lambda_{m}=-\mu_{m}^{2}$. Аналогично доказательству теоремы 1 (а) получим следующий критерий.

Теорема 4. Пусть $-1<k<0$ и $A$ - линейный замкнутый оператор в $E$. Предположим, что граничная задача $(1),(30),(38)$ имеет решение $u(t)$. Для того, чтобы это решение было единственным, необходимо и достаточно, чтобы ни один нуль $\lambda_{m}, m=0,1,2 \ldots$ функции

$$
\Upsilon_{k}^{\alpha, 0}(\lambda)=Y_{k+2}(1 ; \lambda)+\frac{1}{k+1} Y_{k+2}^{\prime}(1 ; \lambda)
$$

не являлся бы собственным значением оператора $A$.

Предыдущие результаты о критерии единственности обобщаются на случай сингулярного уравнения соболевского типа

$$
B\left(u^{\prime \prime}(t)+\frac{k}{t} u^{\prime}(t)\right)=A u(t), \quad 0<t<1
$$

где, также как и $A$, оператор $B$ - линейный замкнутый оператор в $E$, область определения $D(B) \subset E$ которого не обязательно плотна в $E$.

Схема доказательства утверждений аналогична. Отличительная особенность состоит в замене равенства (10) $A h=\lambda h$, определяющего собственные значения оператора $A$, на операторное уравнение $A h=\lambda B h$, если оно имеет нетривиальные решения, а также замене спектра $\sigma_{p}(A)$ на спектр $\sigma_{p}(B, A)$ оператора $A$ относительно $B$. Кроме того, естественно, в определение решения следует дополнительно включить требование принадлежности пространству $C^{2}((0,1), D(B))$.

\section{Список литературы}

[1] Иванов В.К., Мельникова И.В., Филинков А.И. Дифференциально-операторные уравнения и некорректные задачи. М.: Физматлит. 1995.

[2] Кабанихин С.И., Криворотъко О.И. Численный метод решения задачи Дирихле для волнового уравнения // Сиб. журн. индустр. матем. 2012. Т. 15. №4. С. 90 - 101.

[3] Васильев В.И., Кардашевский A.M., Попов В.В. Решение задачи Дирихле для уравнения колебаний струны методом сопряженных градиентов // Вестник СВФУ. 2015 T. 12. №2. С. $43-50$.

[4] Самко С.Г., Килбас А.А., Маричев О.И. Интегралы и производные дробного порядка и некоторые их приложения. Минск, Наука и техника, 1987.

[5] Глушак A.B. О стабилизации решения задачи Дирихле для одного эллиптического уравнения в банаховом пространстве // Дифференц. уравнения. 1997. Т. 33. №4. С. $510-514$. 
[6] Глушак A.B. О разрешимости граничных задач для абстрактного уравнения БесселяСтруве // Дифференц. уравнения. 2019. Т. 55. №8. С. 1103 - 1110.

[7] Глушак А.В., Кононенко В.И., Шмулевич С.Д. Об одной сингулярной абстрактной задаче Коши // Изв. вузов. Матем. 1986. №6. С. 55 - 56.

[8] Ватсон Г.Н. Теория бесселевых функций. М.: ИЛ, 1949.

[9] Боголюбов A.Н., Кравиов В.В. Задачи по математической физике. Учеб. пособие. М.: Изд-во МГУ. 1998.

[10] Керимов М.К. Исследования о нулях специальных функций Бесселя и методах их вычисления // Ж. вычисл. матем. и матем. физ. 2014. Т. 54. №9. С. 1387 - 1441.

[11] Сабитов К.Б. Задача Дирихле для уравнений с частными производными высоких порядков // Матем. заметки. 2015. Т. 97, вып. 2. С. 262-276. 